
ТЕРАПИИ ЛАКТУЛОЗОЙ И ИТОПРИДА ГИДРОХЛОРИДОМ

\author{
Крапивная О.В.1, Алексеенко С.А. ${ }^{2}$ \\ 1 НУз "Дорожная клиническая больница на станции Хабаровск-1 ОАО “РЖД"»; 680022, г. Хабаровск, \\ ул. Воронежская, 49, Российская Федерация \\ 2 ГБОУ ВПО «Дальневосточный государственный медицинский университет» Минздрава России (ГБОУ ВПО \\ ДВГМУ Минздрава России); 680000, г. Хабаровск, ул. Муравьева-Амурского, 35, Российская Федерация
}

Актуальность. В ряде исследований описано частое сочетание гастроэзофагеальной рефлюксной болезни (ГЭРБ), функциональной диспепсии (ФД) и синдрома раздраженного кишечника (СРК).

Цель - изучить особенности клинического течения и диагностики ГЭРБ в сочетании с ФД и СРК с запором (СРК-3) по сравнению с изолированным течением ГЭРБ, а также оценить эффективность терапии лактулозой и итоприда гидрохлоридом. Материал и методы. Обследовано 60 пациентов, страдающих ГЭРБ в сочетании с ФД (Римские критерии III) и СРК-3 (Римские критерии ІІІ) и 29 больных только с ГЭРБ. Диагноз ГЭРБ устанавливали на основании клинических, эндоскопических и рН-метрических критериев. На протяжении 4 недель 10 больных с сочетанием неэрозивной рефлюксной болезни (НЭРБ), ФД и СРК-3 принимали лактулозу, 10 больных - комбинацию лактулозы с итоприда гидрохлоридом. Клиническую

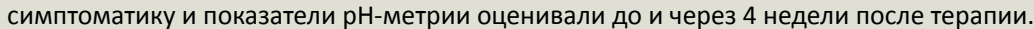

Результаты. Сочетание ГЭРБ, ФД и СРК-З чаще встречалось у женщин моложе 40 лет с нормальным индексом массы тела $(p<0,05)$. Классических симптомов ГЭРБ не отмечалось у 43,4\% больных с коморбидной патологией желудочно-кишечного тракта и у $10,3 \%$ - с изолированным течением ГЭРБ $(p=0,004)$. Распространенность жалоб на отрыжку воздухом и тошноту была выше среди пациентов с сочетанием ГЭРБ, ФД и СРК-3, чем среди больных с изолированным течением ГЭРБ (p<0,05). Через 4 недели лечения у пациентов с сочетанием НЭРБ, ФД и СРК-3, получавших лактулозу в комбинации с итоприда гидрохлоридом, наблюдались уменьшение выраженности клинической симптоматики $(p<0,05)$ и нормализа-

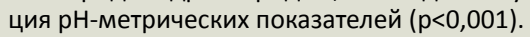

Заключение. Течение ГЭРБ у больных с ФД и СРК-3 сопряжено со следующими особенностями: преобладанием лиц женского пола, отсутствием классических симптомов ГЭРБ почти у половины больных, частым присоединением других функциональных симптомов. Применение комбинации лактулозы и итоприда гидрохлорида позволяет успешно контролировать клинические симптомы ГЭРБ и ФД, а также уровень рН в пищеводе.

Ключевые слова: гастроэзофагеальная рефлюксная болезнь, синдром раздраженного кишечника с запором, функциональная диспепсия.

\title{
GASTROESOPHAGEAL REFLUX DISEASE IN PATIENTS WITH FUNCTIONAL DYSPEPSIA AND CONSTIPATION PREDOMINANT IRRITABLE BOWEL SYNDROME: CLINICAL FEATURES AND EFFICACY OF LACTULOSE AND ITOPRIDE HYDROCHLORIDE
}

\section{Krapivnaya O.V.1, Alekseenko S.A.2}

1 Railroad Clinical Hospital at the Khabarovsk-1 station, JSC RRR, 49 Voronezhskaya ul., Khabarovsk, 680022, Russian Federation

2 Far Eastern State Medical University, 35 Murav'eva-Amurskogo ul., Khabarovsk, 680000, Russian Federation

Background: The frequent coexistence of gastroesophageal reflux disease (GERD) with functional dyspepsia (FD) and an irritable bowel syndrome (IBS) has been described in the literature.

Aim: To study the specific features of GERD clinical course and diagnosis in patients with GERD in combination with FD and constipation predominant IBS (IBS-C) in comparison to patients with isolated GERD; to assess the efficacy of lactulose and itopride hydrochloride.

Materials and methods: A total of 60 patients with GERD, FD (Rome criteria III), and IBS-C (Rome criteria III) and 29 patients with isolated GERD were examined. GERD diagnosis was based on clinical, endoscopic, and pH-metric criteria. For 4 weeks 10 patients 
with combination of non-erosive reflux disease (NERD), FD and IBS-C received lactulose monotherapy and other 10 patients received combination of lactulose with itopride hydrochloride. Clinical symptoms and $\mathrm{pH}$-metric parameters were assessed before and 4 weeks after treatment.

Results: Combination of GERD, FD and IBS-C was noted more frequently in women under 40 with normal body mass index ( $p<0.05$ ). Classic GERD symptoms were absent in $43.4 \%$ of patients with gastrointestinal comorbidity and in $10.3 \%$ of patients with isolated GERD $(p=0.004)$. A higher prevalence of belching and nausea was found in patients, suffering from GERD, FD and IBS-C, than in those with isolated GERD $(p<0.05)$. After 4-week lactulose and itopride hydrochloride treatment all the patients with GERD, FD and IBS-C showed a reduction of clinical symptoms $(p<0.05)$ and normalization of $\mathrm{pH}$-metric parameters $(p<0.001)$. Conclusion: GERD course in patients with concomitant FD and IBS-C has the following peculiarities: predominance of women, absence of classic GERD-symptoms in almost half of these patients, and frequent combination with other functional symptoms. Combination therapy with lactulose and itopride hydrochloride enables successful control of GERD and FD symptoms as well as esophageal pH normalization.

Key words: gastroesophageal reflux disease, irritable bowel syndrome with constipation, functional dyspepsia.

\section{ВВЕДЕНИЕ}

Коморбидность - сосуществование двух или более синдромов или заболеваний у одного пациента, патогенетически взаимосвязанных между собой или совпадающих по времени [1]. Один из примеров коморбидной патологии пищеварительного тракта - сочетание гастроэзофагеальной рефлюксной болезни (ГЭРБ), функциональной диспепсии (ФД) и синдрома раздраженного кишечника (СРК). По данным систематических обзоров, сочетанное течение ГЭРБ встречается у 32-62\% пациентов с ФД [2] и у 11-79\% пациентов с СРК [3]. Сочетанная функциональная патология желудочно-кишечного тракта (ЖКТ) изменяет клиническую картину ГЭРБ [4], что затрудняет дифференциальную диагностику, приводит к неадекватному назначению лекарственной терапии и ее низкой эффективности [5]. Все это отрицательно влияет на качество жизни больных [6].

Клинико-диагностические особенности сочетанного течения ГЭРБ, ФД и СРК с запором (СРК-3) на сегодняшний день не определены, эффективность различных терапевтических подходов изучена недостаточно.

Цель исследования - изучить особенности клинического течения и диагностики ГЭРБ в сочетании с ФД и СРК-3 по сравнению с изолированным течением ГЭРБ, оценить эффективность терапии лактулозой и итоприда гидрохлоридом.

\section{МАТЕРИАЛ И МЕТОДЫ}

Обследовано 60 пациентов, страдающих ГЭРБ в сочетании с ФД и СРК-3 (43 женщины, 17 мужчин, средний возраст - 37,7士1,6 года), и 29 пациентов с изолированным течением ГЭРБ (14 женщин,

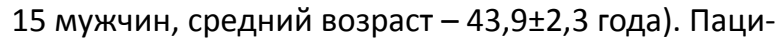
енты включались в исследование по мере обращения в гастроэнтерологическое отделение «Дорожной клинической больницы на станции Хабаровск-1 ОАО “РЖД” » с июня 2012 г. по июнь 2014 г. Диагноз СРК-3 и ФД устанавливали согласно Римским критериям III $[7,8]$, диагноз ГЭРБ - с учетом жалоб пациента, данных эзофагогастродуоденоскопии (ЭГДС) и суточного мониторирования внутрипищеводного pH. Эрозивный эзофагит определяли при наличии эрозивно-язвенного поражения слизистой оболочки пищевода, степень которого оценивали по ЛосАнджелесской классификации [9]. Неэрозивную рефлюксную болезнь (НЭРБ) диагностировали при наличии изжоги или кислой отрыжки 2 и более раза в неделю и при отсутствии видимых повреждений слизистой оболочки пищевода по результатам ЭГДС, а также при наличии патологических изменений, по данным рН-метрии [9]. При включении больного в исследование оценивали такие симптомы, как изжога, кислая отрыжка, тошнота, отрыжка воздухом. Частоту каждого симптома определяли следующим образом: «0» - отсутствие симптома; «1»- симптом отмечался 1 раз в течение недели;

Крапивная Оксана Владимировна - канд. мед. наук, зав. гастроэнтерологическим отделением НУЗ «Дорожная клиническая больница на станции Хабаровск-1 ОАО “РЖД” „. Алексеенко Сергей Алексеевич - д-р мед. наук, профессор, зав. кафедрой госпитальной терапии ГБОУ ВПО ДВГМУ Минздрава России.

Для корреспонденции: Крапивная Оксана Владимировна - 680000, г. Хабаровск, ул. Волочаевская, 163-96, Российская Федерация. Тел.: +7 (4212) 4083 47. E-mail: ovkrapivnaya@gmail.com. Алексеенко Сергей Алексеевич-680000, г. Хабаровск, ул. Муравьева-Амурского, 35, Российская Федерация. Тел.: +7 (4212) 4092 61. E-mail: sa.alexeenko@gmail.com

Krapivnaya Oksana Vladimirovna - MD, PhD, the Head of the Gastroenterological Department, Railroad Clinical Hospital at the Khabarovsk-1 station. Alekseenko Sergey Alekseevich - MD, PhD, Professor, the Head of the Hospital Therapy Chair, Far Eastern State Medical University.

Correspondence to: Krapivnaya Oksana Vladimirovna - 96-163 Volochaevskaya ul., Khabarovsk, 680022, Russian Federation. Tel.: +7 (4212) 4083 47. E-mail: ovkrapivnaya@gmail.com. Alekseenko Sergey Alekseevich - 35 Murav'eva-Amurskogo ul., Khabarovsk, 680000, Russian Federation. Tel.: +7 (4212) 4092 61. E-mail: sa.alexeenko@gmail.com 
Демографические и антропометрические характеристики пациентов с сочетанием ГЭРБ, ФД, СРК-3 и изолированным течением ГЭРБ

\begin{tabular}{c|c|c|c}
\hline Показатель & $\begin{array}{c}\text { Пациенты с сочетанием ГЭРБ, ФД } \\
\text { и СРК-3 (n=60) }\end{array}$ & Пациенты с ГЭРБ (n=29) & p \\
\hline Женщины/мужчины & $43 / 17$ & $13 / 16$ & 0,026 \\
Средний возраст, годы* & $37,7 \pm 1,6$ & $43,9 \pm 2,3$ & 0,030 \\
Индекс массы тела, кг/м² & $24,6 \pm 0,7$ & $28,3 \pm 0,9$ & 0,002 \\
Курение, $\mathrm{n}$ (\%) & $14(23,3)$ & $8(27,6)$ & 0,575 \\
Употребление алкоголя, $\mathrm{n}(\%)$ & $2(3,3)$ & $5(17,2)$ & 0,062
\end{tabular}

* Данные представлены в виде $\mathrm{X} \pm \mathrm{m}_{\mathrm{x}}$.

«2» - симптом наблюдался более 2 раз в неделю; «3» - симптом возникал почти каждый день или ежедневно [10]. Тяжесть симптома оценивали по следующей шкале: «0» - отсутствие симптома; «1» - легкая степень тяжести (симптом проходил самостоятельно); «2» - средняя степень тяжести (симптом самостоятельно не проходил, пациент принимал лекарственный препарат, но эпизодически, воздерживаясь от ежедневного применения); «3» - тяжелая степень (симптом самостоятельно не купировался, требовалось ежедневное применение лекарственного препарата). На основании оценки тяжести и частоты рассчитывали общий балл каждого симптома (от 0 до 6).

Двадцати пациентам с сочетанием НЭРБ, ФД, СРК-3 и 14 - с изолированным течением НЭРБ было выполнено суточное мониторирование внутрипищеводного и внутрижелудочного $\mathrm{pH}$ с помощью аппарата Digitrapper Mk III (Synectics Medical, Швеция). Двадцатичетырехчасовая рН-метрия проводилась с использованием трехканального катетера с расстоянием между электродами 5 см. Катетер располагали таким образом, чтобы электроды находились на 5 см проксимальнее нижнего пищеводного сфинктера, в его зоне и фундальном отделе желудка. После введения катетера в пищевод пациенту рекомендовали вести дневник с указанием времени приема пищи, сна, времени появления симптомов.

Больные с НЭРБ, ФД и СРК-3 были разделены на две группы. Пациенты 1-й группы (n=10) получали лактулозу (Дюфалак, Abbott GmbH \& Co. KG) в индивидуально подобранной дозировке. Пациенты 2-й группы $(\mathrm{n}=10)$ дополнительно к лечению получали итоприда гидрохлорид (Ганатон, Abbott $\mathrm{GmbH} \&$ Co. KG) в дозе 150 мг в день. Исходные характеристики этих групп были одинаковы. До и через 4 недели от начала лечения оценивали клиническую симптоматику СРК и ГЭРБ, показатели рНметрии.

Полученные результаты обрабатывались статистически с определением достоверности различий по t-критерию Стьюдента. В случае повторных измерений использовался парный критерий Стьюдента. Для непараметрических признаков применяли критерий $\chi^{2}$, а при небольшом числе наблюдений - двусторонний вариант точного критерия Фишера. Различия считались достоверными при $p<0,05$. Количественные переменные представлены в работе в виде среднего значения \pm стандартная ошибка среднего $\left(\mathrm{X} \pm \mathrm{m}_{\mathrm{x}}\right)$.

\section{РЕЗУЛЬТАТЫ}

Сочетание ГЭРБ с ФД и СРК-3 достоверно чаще встречалось у лиц женского пола моложе 40 лет с нормальным индексом массы тела $(p<0,05)$. Изолированное течение ГЭРБ чаще наблюдалось у мужчин старше 40 лет с избыточным весом $(p<0,05)$. Никаких существенных различий в других демографических переменных между двумя группами пациентов выявлено не было (табл. 1).

Классические симптомы ГЭРБ (изжога и кислая отрыжка) достоверно чаще встречались у пациентов с изолированным течением ГЭРБ, чем у больных с сочетанием ГЭРБ, ФД, СРК-3 (в 89,7 и 56,6\% случаев соответственно, $p=0,004)$. Распространенность таких функциональных симптомов, как отрыжка воздухом, тошнота, нарушение сна, головная боль, была выше среди пациентов с сочетанием ГЭРБ, ФД, СРК-3, чем среди больных с изолированным течением ГЭРБ, но только для параметров «отрыжка воздухом» и «тошнота» различия достигали статистической значимости ( $<<0,05$, см. рисунок). Доля пациентов с умеренными и тяжелыми классическими симптомами ГЭРБ ( $\geq 5$ баллов в среднем при оценке частоты и тяжести изжоги, кислой отрыжки) существенно не различалась между группами: 16 (61,5\%) из 26 пациентов с изолированной ГЭРБ, 21 (61,7\%) из 34 больных с сочетанием ГЭРБ, ФД, СРК-3 $(p=0,803)$.

По данным эндоскопического исследования, эрозивный эзофагит чаще наблюдался у пациентов 


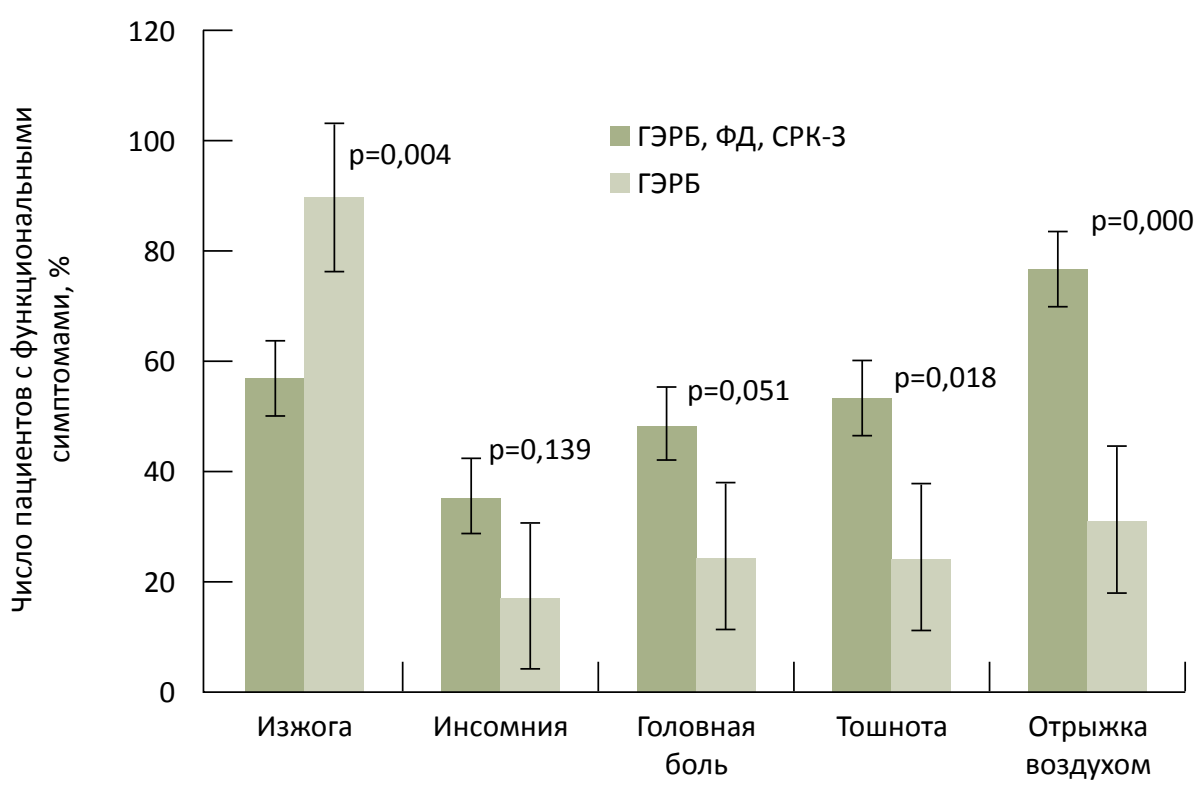

Распространенность клинических симптомов у пациентов с сочетанием ГЭРБ, ФД, СРК-З и изолированным течением ГЭРБ

Примечание: p - достоверность межгрупповых различий.

с изолированным течением ГЭРБ (в 51,7\% случаев), чем с сочетанием ГЭРБ, ФД, СРК-3 (в 30,0\% случаев), однако разница не была статистически значимой ( $p=0,079)$. Среди больных с сочетанной патологией ЖКТ преобладали лица с эзофагитом легкой степени тяжести (эзофагит степени А в соответствии с Лос-Анджелесской классификацией). У пациентов с изолированным течением ГЭРБ эзофагит степени А, В и С диагностирован у пяти, шести и че- тырех человек соответственно (17,2; 20,6 и 13,8\%). Неэрозивная рефлюксная болезнь выявлена у большинства пациентов с сочетанием ГЭРБ, ФД, СРК-3 (70\%).

Двадцати пациентам с сочетанием НЭРБ, ФД, CРК-3 и 14 пациентам с изолированным течением НЭРБ выполнено суточное мониторирование внутрипищеводного рН (табл. 2). Среднее количество кислых и щелочных рефлюксов, а также другие рH-

таблица 2

Результаты суточного мониторирования внутрипищеводного рН у пациентов с сочетанием НЭРБ, ФД, СРК-3 и изолированным течением НЭРБ

\begin{tabular}{|c|c|c|c|}
\hline Показатель, $\mathrm{X} \pm \mathrm{m}_{\mathrm{x}}$ & $\begin{array}{c}\text { Пациенты с сочетанием НЭРБ, ФД, } \\
\text { СРК-3 }(\mathrm{n}=20)\end{array}$ & Пациенты с НЭРБ (n=14) & $\mathrm{p}$ \\
\hline $\begin{array}{l}\text { Среднее время } \\
\text { с внутрипищеводным } \\
\mathrm{pH}<4 / 24 \text { ч, \% }\end{array}$ & $17,8 \pm 4,7$ & $10,2 \pm 3,9$ & 0,252 \\
\hline $\begin{array}{l}\text { Среднее количество кислых } \\
\text { гастроэзофагеальных } \\
\text { рефлюксов }\end{array}$ & $77,4 \pm 6,8$ & $75,8 \pm 5,6$ & 0,866 \\
\hline $\begin{array}{l}\text { Среднее значение индекса } \\
\text { DeMeester/24 ч }\end{array}$ & $58,8 \pm 9,6$ & $33,4 \pm 4,2$ & 0,043 \\
\hline $\begin{array}{l}\text { Среднее значение индекса } \\
\text { симптома, \% }\end{array}$ & $52,8 \pm 9,8$ & $57,9 \pm 10,6$ & 0,731 \\
\hline $\begin{array}{l}\text { Среднее время } \\
\text { с внутрипищеводным } \\
\mathrm{pH}>7 / 24 \text { ч, \% }\end{array}$ & $26,1 \pm 6,7$ & $15,8 \pm 4,6$ & 0,256 \\
\hline $\begin{array}{l}\text { Среднее количество щелочных } \\
\text { рефлюксов }(\mathrm{pH}>7) \\
\text { в пищеводе }\end{array}$ & $29,7 \pm 3,8$ & $34,8 \pm 3,6$ & 0,357 \\
\hline
\end{tabular}


метрические показатели существенно не различались между группами ( $>>0,05)$.

Двадцать пациентов с НЭРБ, ФД, СРК-3 были разделены на две группы, сопоставимые по полу, возрасту, степени тяжести НЭРБ, ФД и СРК-З. У всех больных диагностирован смешанный вариант ФД. Пациенты 1-й группы (n=10) получали лактулозу в средней дозе 40,5 55,7 мл в сутки. Больные из 2-й группы (n=10) принимали комбинацию итоприда гидрохлорида 150 мг в сутки с лактулозой в средней суточной дозе 39,5ะ6,5 мл. Через 4 недели лечения у пациентов обеих групп частота стула повы-

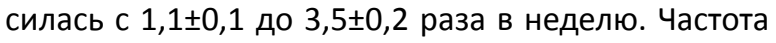
симптомов НЭРБ достоверно уменьшилась во 2-й группе $(p=0,002)$. У всех больных, получавших лечение итоприда гидрохлоридом, и у пяти пациентов 1-й группы купирована симптоматика ФД ( $p=0,035$; точный критерий Фишера). Положительная динамика рН-метрических показателей наблюдалась у пациентов обеих групп, но только у пациентов 2-й группы, получавших комбинацию итоприда гидрохлорида с лактулозой, различия основных показателей суточного мониторирования внутрипищеводного $\mathrm{pH}$ до и после лечения достигали уровня статистической значимости $(p<0,05)$ (табл. 3).

\section{ОБСУЖДЕНИЕ}

По результатам нашего исследования, женщины чаще страдали коморбидной патологией ЖКТ по сравнению с мужчинами. Подобные данные были получены в исследовании S.S. Yarandi и соавт. [11]. Причина высокой распространенности сочетанной функциональной патологии у женщин неизвестна. Обсуждается влияние половых гормонов, гендерных различий в восприятии боли [12].
По нашим данным, высокий индекс массы тела (более $25 \mathrm{kг} / \mathrm{M}^{2}$ ) чаще встречался у больных с изолированным течением ГЭРБ, чем у пациентов с сочетанием ГЭРБ, ФД, СРК-3. Как продемонстрировано в клинических и популяционных исследованиях, ожирение является фактором риска развития ГЭРБ, но не СРК и ФД [13]. Наше исследование не выявило никаких существенных различий в частоте курения или употребления алкоголя между пациентами с изолированным и сочетанным течением ГЭРБ.

Мы изучили частоту таких функциональных симптомов, как тошнота, отрыжка воздухом, головная боль и инсомния, у пациентов с изолированным течением ГЭРБ и сочетанием ГЭРБ, ФД, СРК-3. По нашим данным, частота этих симптомов была выше при сочетанной патологии ЖКТ, однако различия достигали уровня статистической значимости только для параметров «тошнота» и «отрыжка воздухом». Жалобы на тошноту и отрыжку часто встречаются как при ГЭРБ, так и при ФД [14]. Для дифференциальной диагностики НЭРБ с ФД рекомендуется применять суточное мониторирование внутрипищеводного $\mathrm{pH}$ или эмпирическую кислотосупрессивную терапию [14]. По сведениям ряда авторов, жалобы на плохой сон часто наблюдаются и у пациентов с ГЭРБ, и у больных с СРК [15, 16]. Нарушения сна ухудшают течение СРК и являются причиной формирования ГЭРБ, рефрактерной к лечению $[15,16]$. Установлено, что нарушение сна наряду с частой абдоминальной болью повышает вероятность развития ГЭРБ у больных с CPK [13].

В нашем исследовании в структуре ГЭРБ у пациентов с коморбидной патологией ЖКТ доминировала НЭРБ - в 70\% случаев. У больных с изолиро-

таблица 3

Данные суточного мониторирования внутрипищеводного рН у больных 1-й и 2-й групп до и после лечения

\begin{tabular}{|c|c|c|c|c|}
\hline \multirow{2}{*}{ Показатель, X $\pm m_{x}$} & \multicolumn{2}{|c|}{ 1-я группа (n=10) } & \multicolumn{2}{|c|}{ 2-я группа (n=10) } \\
\hline & до лечения & после лечения & до лечения & после лечения \\
\hline $\begin{array}{l}\text { Среднее время с внутрипищеводным } \\
\mathrm{pH}<4 / 24 \text { ч, \% }\end{array}$ & $17,9 \pm 1,7$ & $5,8 \pm 1,4^{* *}$ & $19,7 \pm 2,1^{*}$ & $0,5 \pm 0,1 * * *, * * * *$ \\
\hline $\begin{array}{l}\text { Среднее количество } \\
\text { гастроэзофагеальных рефлюксов }\end{array}$ & $96,0 \pm 18,5$ & $60,5 \pm 11,1^{* *}$ & $109,4 \pm 17,2^{*}$ & $16,1 \pm 4,5 * * *$ \\
\hline $\begin{array}{l}\text { Среднее значение индекса } \\
\text { DeMeester/24 ч }\end{array}$ & $67,1 \pm 8,2$ & $28,3 \pm 1,7^{*}$ & $68,9 \pm 7,2^{*}$ & $1,5 \pm 0,1 * * *, * * * *$ \\
\hline $\begin{array}{l}\text { Среднее значение внутригастрального } \\
\text { рН за период } 24 \text { ч }\end{array}$ & $1,4 \pm 0,2$ & $1,5 \pm 0,3 * *$ & $1,4 \pm 0,1^{*}$ & $2,2 \pm 0,1 * * *, * * * *$ \\
\hline
\end{tabular}

* Достоверность межгрупповых различий до лечения (p>0,1).

** Достоверность различий показателей до и через 4 недели после лечения (p>0,05).

*** Достоверность различий показателей до и через 4 недели после лечения $(p<0,001)$.

**** Достоверность межгрупповых различий после лечения $(p<0,05)$. 
ванным течением ГЭРБ с одинаковой частотой встречались эрозивный эзофагит и НЭРБ (в 44,8 и 55,2\% случаев соответственно). Классические симптомы патологического гастроэзофагеального рефлюкса отсутствовали у $43 \%$ больных с сочетанием ГЭРБ, ФД и СРК-З. Гипотеза о возможном течении ГЭРБ под маской ФД и СРК проверялась в нескольких исследованиях. Y.L. Хіао и соавт. провели суточное мониторирование внутрипищеводного $\mathrm{pH}$ 186 пациентам с клиникой ФД и у $32 \%$ больных выявили патологические изменения, характерные для НЭРБ [17]. Результаты нашего исследования и данные литературы свидетельствуют о трудностях диагностики ГЭРБ у больных с функциональной патологией жКТ. В сложных случаях необходимо проводить суточную рН-метрию.

Алгоритм лечения ГЭРБ у больных с сочетанием ФД и СРК-3 не разработан. Этой проблеме были посвящены недавно проведенные исследования H. Mönnikes и J.C.Y. Wu. В работе H. Mönnikes приняли участие 1888 больных с ГЭРБ, среди них у 15\% наблюдались СРК-подобные симптомы [18]. Пациенты получали 40 мг пантопразола на протяжении 8 недель. Эффективность лечения пантопразолом через 4 и 8 недель была ниже у пациентов с сочетанием ГЭРБ и СРК, чем у страдающих изолированной ГЭРБ (p<0,05). Авторы пришли к выводу, что наличие СРК-подобных симптомов отрицательно влияет на результаты лечения ГЭРБ. В исследовании J.C.Y. Wu изучалась эффективность приема ингибиторов протонной помпы «по требованию» у больных с разными формами ГЭРБ [19]. Сопутствующие ФД и СРК были двумя независимыми прогностическими факторами неэффективности терапии ГЭРБ «по требованию».

Тактика ведения пациентов с сочетанием ГЭРБ и функциональных заболеваний ЖКТ в случае низкой эффективности ингибиторов протонной помпы не определена. В нашем исследовании мы назнача-

\section{Литература}

1. Стяжкина СН, Журавлев КВ, Леднева АВ, Ларин ВВ, Климентов МН, Чернышева ТЕ. Роль коморбидной патологии в хирургии. Фундаментальные исследования. 2011;(7):138-40. (Styazhkina SN, Zhuravlev KV, Ledneva AV, Larin VV, Klimentov MN, Chernysheva TE. [Role of comorbid pathology in surgery]. Fundamental'nye issledovaniya. 2011;(7):138-40. Russian).

2. Keohane J, Quigley EM. Functional dyspepsia and nonerosive reflux disease: clinical interactions and their implications. MedGenMed. 2007;9(3):31.

3. De Bortoli N, Martinucci I, Bellini M, Savarino E, Savarino V, Blandizzi C, Marchi S. Overlap of functional heartburn and gastroesophageal reflux disease with irritable bowel syndrome. World J Gastroenterol. 2013;19(35):5787-97. ли пациентам данной группы прокинетик итоприда гидрохлорид в комбинации с лактулозой. Полученные результаты свидетельствуют о том, что с помощью итоприда гидрохлорида можно успешно контролировать клинические симптомы НЭРБ и ФД у больных с сочетанной функциональной патологией, а также уровень рН в пищеводе, количество гастроэзофагеальных рефлюксов. Как продемонстрировано в экспериментальных и клинических исследованиях, итоприда гидрохлорид усиливает пропульсивную моторику пищевода и желудка и ускоряет опорожнение желудка [20].

По результатам российского многоцентрового клинического исследования было отмечено, что у больных с изжогой применение итоприда гидрохлорида в течение 4 недель приводило к полному устранению симптома в 60,4\%, значительному уменьшению выраженности изжоги - в 27,8\%. Отрыжка исчезла полностью у 48,1\% больных, значительно уменьшилась у 34,7\%, регургитация (срыгивание) - у 88,1 и 11,9\% соответственно [21].

\section{ВЫводы}

1. Особенности течения ГЭРБ у больных с ФД и СРК-3 связаны с отсутствием классических симптомов ГЭРБ почти в половине случаев, частым сочетанием с такими функциональными симптомами, как отрыжка воздухом, тошнота, нарушение сна, головная боль, высокой распространенностью неэрозивной формы ГЭРБ.

2. Сочетанное течение ГЭРБ с ФД и СРК чаще отмечается у женщин моложе 40 лет с нормальным индексом массы тела.

3. Применение комбинации лактулозы (Дюфалака) и итоприда гидрохлорида (Ганатона) приводит к нормализации стула, купированию проявлений ГЭРБ и ФД, уменьшению показателей кислотной экспозиции в пищеводе у больных с сочетанием ГЭРБ и СРК-3.

4. Крапивная ОВ, Алексеенко СА. Опыт применения омепразола быстрого высвобождения при гастроэзофагеальной рефлюксной болезни с функциональной диспепсией и с синдромом раздраженного кишечника с запором. Клинические перспективы гастроэнтерологии, гепатологии. 2014;(2): 25-9. (Krapivnaya OV, Alekseenko SA. [Application of immediate-release omeprazole in the gastroesophageal reflux disease with functional dyspepsia and constipation predominant irritable bowel syndrome]. Klinicheskie perspektivy gastroenterologii, gepatologii. 2014;(2):25-9. Russian).

5. Sifrim D, Zerbib F. Diagnosis and management of patients with reflux symptoms refractory to proton pump inhibitors. Gut. 2012;61(9):1340-54. 
6. Kaji M, Fujiwara Y, Shiba M, Kohata Y, Yamagami H, Tanigawa T, Watanabe K, Watanabe T, Tominaga K, Arakawa T. Prevalence of overlaps between GERD, FD and IBS and impact on health-related quality of life. J Gastroenterol Hepatol. 2010;25(6):1151-6.

7. Longstreth GF, Thompson WG, Chey WD, Houghton LA, Mearin F, Spiller RC. Functional bowel disorders. Gastroenterology. 2006;130(5):1480-91.

8. Алексеенко СА, Логинов АФ, Крапивная ОВ, Николаева АЕ. Соответствие Римских критериев II и III в диагностике функциональной диспепсии и сравнительная оценка эффективности ее лечения малыми дозами фамотидина. Клинические перспективы гастроэнтерологии, гепатологии. 2006;(5): 25-7. (Alekseenko SA, Loginov AF, Krapivnaya OV, Nikolaeva AE. [The Rome II and III criteria in diagnostic management of functional dyspepsia and comparative assessment of its treatment with low dose famotidin]. Klinicheskie perspektivy gastroenterologii, gepatologii. 2006;(5):25-7. Russian).

9. Алексеенко СА. Алгоритмы диагностики и лечения гастроэзофагеальной рефлюксной болезни. Фарматека. 2006;(1): 48-9. (Alekseenko SA. [Algorithm for diagnosis and treatment of gastroesophageal reflux disease]. Farmateka. 2006;(1):48-9. Russian).

10. Bennett EJ, Tennant CC, Piesse C, Badcock CA, Kellow JE. Level of chronic life stress predicts clinical outcome in irritable bowel syndrome. Gut. 1998;43(2):256-61.

11. Yarandi SS, Nasseri-Moghaddam S, Mostajabi P, Malekzadeh R. Overlapping gastroesophageal reflux disease and irritable bowel syndrome: increased dysfunctional symptoms. World J Gastroenterol. 2010;16(10):1232-8.

12. Frissora $\mathrm{CL}$, Koch $\mathrm{KL}$. The role of gender and biological sex in irritable bowel syndrome. Curr Gastroenterol Rep. 2005;7(4):257-63.

13. Jung HK, Halder $S$, McNally $M$, Locke GR $3^{\text {rd }}$, Schleck $C D$, Zinsmeister AR, Talley NJ. Overlap of gastro-oesophageal reflux disease and irritable bowel syndrome: prevalence and risk factors in the general population. Aliment Pharmacol Ther. 2007;26(3):453-61.
14. Tack J, Talley NJ, Camilleri M, Holtmann G, Hu P, Malagelada JR, Stanghellini V. Functional gastroduodenal disorders. Gastroenterology. 2006;130(5):1466-79.

15. Bellini M, Gemignani A, Gambaccini D, Toti S, Menicucci D, Stasi C, Costa F, Mumolo MG, Ricchiuti A, Bedini R, de Bortoli N, Marchi S. Evaluation of latent links between irritable bowel syndrome and sleep quality. World J Gastroenterol. 2011;17(46): 5089-96.

16. Fujiwara Y, Arakawa T, Fass R. Gastroesophageal reflux disease and sleep disturbances. J Gastroenterol. 2012;47(7):760-9.

17. Xiao YL, Peng S, Tao J, Wang AJ, Lin JK, Hu PJ, Chen MH. Prevalence and symptom pattern of pathologic esophageal acid reflux in patients with functional dyspepsia based on the Rome III criteria. Am J Gastroenterol. 2010;105(12):2626-31.

18. Mönnikes $H$, Heading RC, Schmitt $H$, Doerfler $H$. Influence of irritable bowel syndrome on treatment outcome in gastroesophageal reflux disease. World J Gastroenterol. 2011;17(27): 3235-41.

19. Wu JC, Lai LH, Chow DK, Wong GL, Sung JJ, Chan FK. Concomitant irritable bowel syndrome is associated with failure of stepdown on-demand proton pump inhibitor treatment in patients with gastro-esophageal reflux disease. Neurogastroenterol Motil. 2011;23(2):155-60.

20. Scarpellini E, Vos R, Blondeau K, Boecxstaens V, Farré R, Gasbarrini A, Tack J. The effects of itopride on oesophageal motility and lower oesophageal sphincter function in man. Aliment Pharmacol Ther. 2011;33(1):99-105.

21. Ивашкин ВТ, Шептулин АА, Трухманов АС. Клинические аспекты функциональной диспепсии и эффективность ганатона (итоприда гидрохлорида) в ее лечении. Российский журнал гастроэнтерологии, гепатологии, колопроктологии. 2009;(6):17-22. (Ivashkin VT, Sheptulin AA, Trukhmanov AS. [Clinical aspects of functional dyspepsia and efficacy of ganaton (itopride hydrochloride]. Rossiyskiy zhurnal gastroenterologii, gepatologii, koloproktologii. 2009;(6): 17-22. Russian). 